\title{
POINTWISE BOUNDS FOR SOLUTIONS OF A CLASS OF FUNCTIONAL EQUATIONS*
}

BY

S. C. CHU' (University of Delaware) AND F. T. METCALF² (University of California, Riverside)

1. Introduction. The purpose of this paper is to present an approach with which one can, in a simple way, obtain point-wise bounds for solutions of certain types of equations. Consider, for example, the linear Volterra integral equation of the second kind

$$
u(x)=f(x)+\int_{0}^{x} k(x, y) u(y) d y, \quad 0 \leq x \leq 1,
$$

where, for simplicity, it is assumed that $f$ is real and continuous on $0 \leq x \leq 1$, and $k$ is real and continuous on $0 \leq y \leq x \leq 1$. The solution $u$, then, satisfies

$$
|u(x)-f(x)| \leq \int_{0}^{x}|k(x, y)| \cdot|u(y)| d y, \quad 0 \leq x \leq 1,
$$

and hence,

$$
|u(x)-f(x)| \leq \int_{0}^{x}|k(x, y)| M(y) d y, \quad 0 \leq x \leq 1,
$$

where $M(y)=\sup _{0 \leq z \leq v}|u(z)|$. If, now, one can find an upper bound for $M$, in terms of the given functions $f$ and $k$, then (2) gives an upper bound for $|u(x)-f(x)|$, and hence, both upper and lower point-wise bounds for $u$.

It follows from (2) that

$$
|u(z)| \leq|f(z)|+M(z) \int_{0}^{z}|k(z, y)| d y, \quad 0 \leq z \leq 1,
$$

and hence,

$$
\sup _{0 \leq z \leq x}|u(z)| \leq \sup _{0 \leq z \leq x}|f(z)|+\sup _{0 \leq z \leq x}\left[M(z) \int_{0}^{z}|k(z, y)| d y\right], \quad 0 \leq x \leq 1 .
$$

This, in turn, gives

$$
M(x) \leq M_{f}(x)+M(x) \sup _{0 \leq z \leq x} \int_{0}^{z}\left|l_{i}(z, y)\right| d y,
$$

where $M_{f}(x)=\sup _{0 \leq x \leq x}|f(z)|$. If, in addition, one assumes that

$$
\sup _{0 \leq z \leq x} \int_{0}^{z}\left|l_{i}(z, y)\right| d y<1
$$

*Received November 21, 1966.

${ }^{1}$ The research of this author was performed while he was at Bellcomm, Inc., Washington, D. C.

${ }^{2}$ The research of this author was performed while he was at the Institute for Fluid Dynamics and Applied Mathematics, University of Maryland, College Park, Maryland, and supported, in part, by the Air Force Office of Scientific Research, Grant AFOSlR 400-6J and 1122-67. 
for $0 \leq x \leq 1$, then

$$
M(x) \leq M_{f}(x)\left[1-\sup _{0 \leq x \leq x} \int_{0}^{z}|k(z, y)| d y\right]^{-1}, \quad 0 \leq x \leq 1 .
$$

Thus, the right-hand side of this last inequality serves as the desired upper bound for $M$, and yields, upon substitution into (2),

$$
|u(x)-f(x)| \leq \int_{0}^{x}|k(x, y)| M_{f}(y)\left[1-\sup _{0 \leq z \leq \nu} \int_{0}^{z}|k(z, w)| d w\right]^{-1} d y,
$$

for all $0 \leq x \leq 1$. In order to avoid integration, the right-hand side of (3) may be simplified by replacing the quantities

$$
\sup _{0 \leq z \leq \nu} \int_{0}^{z}|k(z, w)| d w, \quad 0 \leq y \leq x \leq 1,
$$

and

$$
\int_{0}^{x}|k(x, y)| d y, \quad 0 \leq x \leq 1
$$

with

$$
x \sup _{0 \leq v \leq z \leq x}|k(z, y)|, \quad 0 \leq x \leq 1
$$

and

$$
x \sup _{0 \leq \nu \leq x}|k(x, y)|, \quad 0 \leq x \leq 1,
$$

respectively (under the restriction, of course, that

$$
x \sup _{0 \leq \nu \leq z \leq x}|k(z, y)|<1
$$

for $0 \leq x \leq 1)$. The bound on $|u(x)-f(x)|$ would then become

$$
|u(x)-f(x)| \leq x M I_{f}(x) \sup _{0 \leq \nu \leq x}|k(x, y)| \cdot\left[1-x \sup _{0 \leq \nu \leq s \leq x}|k(z, y)|\right]^{-1},
$$

for all $0 \leq x \leq 1$.

The foregoing, then, gives point-wise bounds for the solution of Eq. (1) with very little work. (This was also essentially the method used by Balachandran [1] to obtain bounds for solutions of Fredholm integral equations of the second kind.) However, it has the great disadvantage that one must assume that

$$
\sup _{0 \leq x \leq 1} \int_{0}^{z}|k(z, y)| d y<1 .
$$

If this restriction on the kernel $k$ is not satisfied, then what would normally have to be done is to iterate Eq. (1) for a sufficiently large number of times, until some iterated kernel satisfies that condition. But this procedure would involve finding the iterated kernels, which, in general, is not easy. It is, therefore, desirable to find a method for obtaining bounds of the type (3) or (4), which retains the simplicity of the procedure described above, without performing iterations, and yet not requiring any restrictions about the "size" of the kernel $k$. 
In Sec. 2, the foregoing simple procedure (together with the "size" restriction) is stated in general terms, thereby, permitting application to other types of equations. A way is then indicated for circumventing the "size" restriction for a certain class of equations. In Sec. 3, this combined procedure is applied to various problems. Point-wise bounds are obtained for solutions of integral equations, functional equations, and functional-integral equations.

2. The method. The simple technique, briefly described in Sec. 1, can be put in a more general form. Let $S$ be a subset of the real numbers $R$. Let $C$ be the class of functions defined by

$$
C=\left\{v \mid v: S \rightarrow R, \quad v \text { continuous on } S, \sup _{x \varepsilon s}|v(x)|<\infty\right\} .
$$

(By continuity, here, is meant the usual " $\epsilon, \delta$ " definition; that is, $v$ is continuous at $x \in S$ if, for every $\epsilon>0$, there exists a $\delta>0$ such that $|x-y|<\delta, y \in S$, implies $|v(x)-v(y)|<\epsilon$. This definition permits $S$ to have isolated points.) Consider the functional equation

$$
u(x)=f(x)+T\left(x ; u(s), s \in S_{0}(x)\right), \quad x \in S,
$$

where $f \in C, S_{0}(x)$ is a subset of $S$ depending on $x \in S$, and $T$ is a mapping taking $S \times C$ into $R$, and is sometimes written $T=T(x ; u(\cdot))$, for convenience. It is assumed that the mapping $T$ is such that, there exists a mapping $\tilde{T}$ of $S \times C$ into $R$, satisfying:

(i) $|T(x ; u(\cdot))| \leq \tilde{T}(x ;|u|(\cdot))$ for all $x \in S, u \in C$;

(ii) $u_{1} \in C, u_{2} \in C$, and $0 \leq u_{1}(s) \leq u_{2}(s)$ for $s \in S_{0}(x)$, imply $\tilde{T}\left(x ; u_{1}(\cdot)\right) \leq$ $\tilde{T}\left(x ; u_{2}(\cdot)\right)$, for all $x \in S$

(iii) there exists a $g \in C$ such that $\tilde{T}(x ; u(\cdot)) \leq g(x) \sup _{\nu \in s_{0}(x)} u(y)$, for any $u \in C$ such that $0 \leq u(x), x \in S$.

Let $S_{1}(x), x \in S$, be a family of sets with the properties:

(iv) $x \in S_{1}(x)$ for all $x \in S$;

(v) $S_{0}(x) \subseteq S_{1}(x) \subseteq S$ for all $x \in S$;

(vi) $S_{1}(y) \subseteq S_{1}(x)$ for each $y \in S_{1}(x), x \in S$;

(vii) $u \in C, 0 \leq u(x)$ for all $x \in S$, imply $v(x)=\sup _{y \in s_{1}(x)} u(y), x \in S$, is in $C$.

Such sets certainly exist, since the set $S$ itself has these properties. (The forms of both $S_{0}(x)$ and $S_{1}(x)$, for specific problems, will be given in the applications of the next section.)

Theorem 1. Suppose Eq. (5) possesses a solution $u \in C$. Suppose the mapping $T$ is such that there exists a $\tilde{T}$ satisfying the conditions (i)-(iii) above, and, in addition,

$$
\sup _{\boldsymbol{y} \in S_{1}(x)} g(y)<1, \quad \text { for all } x \in S,
$$

where $S_{1}(x), x \in S$, is a family of sets satisfying (iv)-(vii). Then the function u has the pointwise bounds given by

$$
|u(x)-f(x)| \leq \tilde{T}(x ; U(\cdot)), \quad x \in S,
$$

where

$$
U(x)=\sup _{y \in S_{1}(x)}|f(y)| /\left(1-\sup _{y \in S_{1}(x)} g(y)\right), \quad x \in S .
$$


Proof. Let

$$
M(x)=\sup _{\nu \in S_{1}(x)}|u(y)|
$$

for $x \in S$. Condition (iv) above gives that $|u(x)| \leq M(x)$ for each $x \in S$. Hence, from Fq. (5):

$$
\begin{aligned}
& |u(x)-f(x)| \leq|T(x ; u(\cdot))| \leq \tilde{T}(x ;|u|(\cdot)), \quad x \in S ; \\
& |u(x)-f(x)| \leq \tilde{T}(x ; M(\cdot)), \quad x \in S,
\end{aligned}
$$

where use is made of condition (ii) and the fact that $M \in C$ (condition (vii)). Conditions (v) and (vi) allow one to conclude that

$$
M(s) \leq M(x), \quad s \in S_{0}(x), \quad x \in S ;
$$

and this gives, using property (iii),

$$
\tilde{T}(x ; M(\cdot)) \leq g(x) \sup _{s \in S_{0}(x)} M(s) \leq g(x) M(x), \quad x \in S .
$$

Inequality (7) then yields, for every $x \in S$,

$$
|u(x)| \leq|f(x)|+g(x) M(x) .
$$

Therefore, upon taking the supremum of both sides (over the set $S_{1}(x)$ ), and noting that condition (vi) gives $\sup _{\nu e S_{1}(x)} M(y) \leq M(x)$, one obtains

$$
M I(x) \leq \sup _{\boldsymbol{y} \in S_{\mathbf{1}}(x)}|f(y)|+M(x) \sup _{\boldsymbol{y} \in S_{\mathbf{1}}(x)} g(y), \quad x \in S
$$

which may be solved for $M(x)$ :

$$
M(x) \leq \frac{\sup _{y \in S_{1}(x)}|f(y)|}{1-\sup _{\boldsymbol{y} \in S_{1}(x)} g(y)}=U(x), \quad x \in S .
$$

This, together with (7), gives the desired result, after noting that $U \in C$.

REMARKs. At the expense of "iterating" the mapping $\tilde{T}$, one can obtain a sequence of decreasing upper bounds in the following manner. Let

$$
U_{n+1}(x)=|f(x)|+\tilde{T}\left(x ; U_{n}(\cdot)\right), \quad x \in S,
$$

for $n=0,1,2, \cdots$, where $U_{0}=U$. It follows from the result of Theorem 1 that

$$
|u(x)| \leq|f(x)|+\tilde{T}(x ; U(\cdot))=U_{1}(x), \quad x \in S .
$$

Hence, from (6), one has

$$
|u(x)-f(x)| \leq \tilde{T}\left(x ; U_{1}(\cdot)\right), \quad x \in S .
$$

By induction, it readily follows that

$$
|u(x)-f(x)| \leq \tilde{T}\left(x ; U_{n}(\cdot)\right), \quad x \in S,
$$

for $n=0,1,2, \cdots$. Furthermore,

$$
\begin{aligned}
U_{1}(x) & \leq|f(x)|+\tilde{T}(x ; \bar{U}(x)(\cdot)) \\
& \leq|f(x)|+\bar{U}(x) g(x) \\
& \leq \sup _{\boldsymbol{y} \in S_{1}(x)}|f(y)|+\bar{U}(x) \sup _{\boldsymbol{y} \in S_{2}(x)} g(y),
\end{aligned}
$$


for all $x \in S$, where

$$
\begin{aligned}
\bar{U}(x) & =\sup _{s \in S_{0}(x)} U(s) \\
& =\sup _{\boldsymbol{s} \in S_{0}(x)}\left[\sup _{\boldsymbol{\nu} \in S_{1}(s)}|f(y)| \cdot 1 /\left(1-\sup _{\boldsymbol{y} \in S_{\mathbf{1}}(s)} g(y)\right)\right] \\
& \leq \sup _{\boldsymbol{\nu} \in S_{\mathbf{1}}(x)}|f(y)| \cdot 1 /\left(1-\sup _{\boldsymbol{y} \in S_{1}(x)} g(y)\right)=U(x),
\end{aligned}
$$

for all $x \in S$; and hence,

$$
U_{1}(x) \leq \sup _{\boldsymbol{y} \in S_{1}(x)}|f(y)|+U(x) \sup _{\boldsymbol{y} \in S_{1}(x)} g(y)=U(x),
$$

for all $x \in S$. Induction may now be used to give

$$
U_{n+1}(x) \leq U_{n}(x), \quad x \in S,
$$

for each $n=0,1,2, \cdots$.

Although, for simplicity, Theorem 1 was stated for functions of class $C$, similar results, by analogous reasoning, can be obtained for other function classes. Balachandran [1], for example, applied the same type of reasoning to the specific problem of finding point-wise bounds for functions which are square-integrable and which satisfy linear Fredholm integral equations of the second kind.

Theorem 1, then, provides a very simple method for obtaining point-wise bounds for solutions of equations of the type (5). It has the disadvantage, however, that the mapping $T$ must be such that

$$
\sup _{y \in S_{1}(x)} g(y)<1,
$$

for all $x \in S$; that is, the "size" of $T$ is restricted.

A way will now be indicated, whereby, for certain types of equations, the "size" restriction can be circumvented and yet the simple procedure described above can be retained. This is accomplished by, essentially, a change of the dependent variable. Let $K$ be a linear, invertible mapping of $C$ into $C$; suppose $K$ is order-preserving in the sense that $0 \leq u(x), x \in S$, implies that $0 \leq K u(x)$. Then, if $u$ is a solution of Tq. (5), $u$ must satisfy

$$
K K^{-1} u(x)=f(x)+T\left(x ; K K^{-1} u(s), s \in S_{0}(x)\right), \quad x \in S .
$$

Define $v(x)=K^{-1} u(x)$ for $x \in S$. Then $v$ satisfies

$$
v(x)=K^{-1} f(x)+K^{-1} T\left(x ; K v(s), s \in S_{0}(x)\right), \quad x \in S ;
$$

i.e., $v$ satisfies the equation

$$
v(x)=f_{1}(x)+T_{1}(x ; v(\cdot)), \quad x \in S,
$$

where $f_{1}=K^{-\cdots} f$ and $T_{1}(x ; v(\cdot))=K^{-1} T(x ; K v(\cdot))$ for all $x \in S$. For certain equations, with a suitable choice of $K$, the mapping $T_{1}$ may satisfy all the hypotheses of Theorem 1 , although the original mapping $T$ may not. In such cases, one merely obtains first the point-wise bounds for the function $v$, and then makes the substitution $u=K v$. The order-preserving feature of $K$ will then yield point-wise bounds for the function $u$. This approach will now be made clearer by means of the following applications. 


\section{Applications.}

(a) Volterra Integral Equations of the Second Kind. Consider, again, Eq. (1). In this case $S=[0,1], S_{0}(x)=S_{1}(x)=[0, x]$,

$$
\begin{array}{ll}
T(x ; u(\cdot))=\int_{0}^{x} k(x, y) u(y) d y, & 0 \leq x \leq 1, \\
\tilde{T}(x ; u(\cdot))=\int_{0}^{x}|k(x, y)| u(y) d y, & 0 \leq x \leq 1,
\end{array}
$$

and

$$
g(x)=\int_{0}^{x}|k(x, y)| d y, \quad 0 \leq x \leq 1 .
$$

A direct application of Theorem 1 would, as noted in Sec. 1, yield bounds for the solution $u$, provided that the kernel satisfies the restriction

$$
\sup _{0 \leq s \leq 1} \int_{0}^{z}|k(z, y)| d y<1 .
$$

If the kernel $k$ does not satisfy this condition, then one may consider, instead, the equation satisfied by the function $v(x)=K^{-1} u(x)=e^{-\lambda x} u(x), 0 \leq x \leq 1$, where $\lambda>0$; that is, the equation

$$
v(x)=f(x) e^{-\lambda x}+e^{-\lambda x} \int_{0}^{x} k(x, y) e^{\lambda y} v(y) d y, \quad 0 \leq x \leq 1 .
$$

Setting

$$
\tilde{T}_{1}(x ; v(\cdot))=e^{-\lambda x} \int_{0}^{x}|k(x, y)| e^{\lambda y} v(y) d y, \quad 0 \leq x \leq 1,
$$

one has that $\tilde{T}_{1}$ satisfies the "size" hypothesis of Theorem 1, provided

$$
\sup _{0 \leq s \leq 1} e^{-\lambda s} \int_{0}^{z}|k(z, y)| e^{\lambda_{y}} d y<1 .
$$

But this condition is automatically satisfied for $\lambda$ sufficiently large, since, for $0 \leq z \leq 1$,

$$
\begin{aligned}
e^{-\lambda_{s}} \int_{0}^{z}|k(z, y)| e^{\lambda_{y}} d y & \leq e^{-\lambda_{z}} \int_{0}^{z} e^{\lambda_{y}} d y \cdot \sup _{0 \leq y \leq z}|k(z, y)| \\
& \leq \frac{1-e^{-\lambda_{z}}}{\lambda} \cdot \sup _{0 \leq y \leq z \leq 1}|k(z, y)| \\
& \leq \frac{1-e^{-\lambda}}{\lambda} \cdot \sup _{0 \leq y \leq z \leq 1}|k(z, y)|<1,
\end{aligned}
$$

for all $\lambda$ such that

$$
\sup _{0 \leq \nu \leq z \leq 1}|k(z, y)|<\frac{\lambda}{1-e^{-\lambda}} .
$$

Hence, application of Theorem 1 yields

$$
\left|v(x)-f(x) e^{-\lambda x}\right| \leq e^{-\lambda x} \int_{0}^{x}|k(x, y)| e^{\lambda y} V(y) d y, \quad 0 \leq x \leq 1,
$$


where

$$
V(y)=\frac{\sup _{0 \leq z \leq \nu}|f(z)| e^{-\lambda z}}{1-\sup _{0 \leq z \leq \nu} e^{-\lambda z} \int_{0}^{z}|k(z, t)| e^{\lambda t} d t}, \quad 0 \leq y \leq 1 .
$$

Recalling that $u(x)=v(x) e^{\lambda x}$, one obtains the following:

Theorem 2. Let $f$ and $k$ be real and continuous on $0 \leq x \leq 1$ and $0 \leq y \leq x \leq 1$, respectively. Let $u$ be a real and continuous solution of the equation

$$
u(x)=f(x)+\int_{0}^{x} k(x, y) u(y) d y, \quad 0 \leq x \leq 1
$$

Then,

$$
|u(x)-f(x)| \leq \int_{0}^{x}|k(x, y)| e^{\lambda y} V(y) d y, \quad 0 \leq x \leq 1,
$$

where $V$ is defined by (9),

and $\lambda$ satisfies

$$
\lambda=0, \quad \text { if } \sup _{0 \leq z \leq 1} \int_{0}^{z}|k(z, y)| d y<1,
$$

$$
\frac{\lambda}{1-e^{-\lambda}}>\sup _{0 \leq y \leq z \leq 1}|k(z, y)|, \quad \text { if } \sup _{0 \leq z \leq 1} \int_{0}^{z}|k(z, y)| d y \geq 1 .
$$

REMARKs. It is clear that a better estimate for $u$ can be obtained by, instead of bounding the quantity $|u(x)-f(x)|$, bounding the quantity

$$
\left|u(x)-f(x)-\int_{0}^{x} k(x, y) f(y) d y-\cdots-\int_{0}^{x} k_{n}(x, y) f(y) d y\right|,
$$

for some positive integer $n$, where $k_{i}(i=1, \cdots, n)$ is the $i$ th iterated kernel. However, this procedure involves iterated integration, and the main purpose in this paper is to give a simple and quick way of obtaining point-wise bounds, without any repeated integration.

The procedure applies equally well if one considers the space of square-integrable functions instead of the continuous functions, even though Theorem 1 cannot be directly applied. For the square-integrable case, again consider the associated equation for $v$, namely, Eq. (8). Then, by applying a computation procedure similar to that used by Balachandran [1] for Fredholm equations, one obtains the estimate

$$
|u(x)-f(x)| \leq\left[\int_{0}^{x} e^{2 \lambda{ }^{2}} k^{2}(x, y) d y\right]^{1 / 2} \frac{b(x)+\left[b^{2}(x)-a(x) c(x)\right]^{1 / 2}}{a(x)},
$$

for $0 \leq x \leq 1$, where

$$
\begin{aligned}
& a(x)=1-\int_{0}^{x} \int_{0}^{y} k^{2}(y, z) e^{-2 \lambda(y-z)} d z d y, \\
& b(x)=\left[\int_{0}^{x}\left(\int_{z}^{x} k(y, z) f(y) e^{-\lambda(2 y-z)} d y\right)^{2} d z\right]^{1 / 2}, \\
& c(x)=\int_{0}^{x} f^{2}(z) e^{-2 \lambda z} d z,
\end{aligned}
$$

and $\lambda$ is chosen such that $a(x)>0$ for all $0 \leq x \leq 1$. 
It may be observed here that, in both the continuous case and the square-integrable case, the above bounds yield, immediately, the uniqueness of a solution to Eq. (1), since if $u_{1}$ and $u_{2}$ are solutions of (1), then $u_{1}-u_{2}$ is a solution of (1) with $f \equiv 0$.

Finally, it should be remarked that the method described here can be applied to certain nonlinear equations (where the integral is of the form

$$
\int_{0}^{x} k(x, y, u(y)) d y
$$

with $k$ satisfying some kind of Lipschitz condition), to systems of equations of the type (1) or their nonlinear analogues, to equations (and systems) in several variables of the form

$$
\begin{aligned}
u(x, y)=f(x, y)+ & \int_{0}^{x} k_{1}(x, y ; z) u(z, y) d z \\
& \quad+\int_{0}^{\nu} k_{2}(x, y ; z) u(x, z) d z+\int_{0}^{x} \int_{0}^{y} k_{3}\left(x, y ; z_{1}, z_{2}\right) u\left(z_{1}, z_{2}\right) d z_{1} d z_{2},
\end{aligned}
$$

and to certain nonlinear versions of such.

(b) Functional Equations. Consider the functional equation

$$
u(x)=f(x)+g(x) u(\alpha(x)), \quad 0 \leq x \leq 1,
$$

where $f, g$, and $\alpha$ are real and continuous on $0 \leq x \leq 1 ; \alpha(0)=0,0 \leq \alpha(x)<x$ for $0<x \leq 1$; and $|g(0)|<1$. Under these circumstances one has the existence of a continuous solution $u$, see, for example, Chu and Diaz [2]. In this case, one may take $S=[0,1], S_{0}(x)=\{\alpha(x)\}, S_{1}(x)=[0, x], T(x, u(\alpha(x)))=g(x) u(\alpha(x))$, and $K^{-1} u(x)=$ $e^{-\lambda x} u(x)$, for $0 \leq x \leq 1$ and $\lambda>0$. Then, the function $v(x)=e^{-\lambda x} u(x)$ satisfies the equation

$$
v(x)=e^{-\lambda x} f(x)+g(x) \exp (-\lambda(x-\alpha(x))) v(\alpha(x)), \quad 0 \leq x \leq 1 .
$$

Theorem 1 can be applied to obtain a bound on $v$, provided that

$$
\sup _{0 \leq \nu \leq x}|g(y) \exp (-\lambda(y-\alpha(y)))|<1, \quad 0 \leq x \leq 1 .
$$

But, for $\lambda$ sufficiently large, this is indeed the case. Since $g$ is continuous and $|g(0)|<1$, there exists a $0<\delta \leq 1$ such that, for $0 \leq x \leq \delta$, one has

$$
|g(x) \exp (-\lambda(x-\alpha(x)))|<1,
$$

for any $\lambda \infty 0$. For $\delta \leq x \leq 1$, the quantity $|g(x) \exp (-\lambda(x-\alpha(x)))|$ can be made as small as desired by simply choosing $\lambda$ sufficiently large. In particular, let $m=$ $\min _{\delta \leq y \leq 1}(y-\alpha(y))$, and choose $\lambda$ such that

$$
\lambda>\frac{1}{m} \log \left[\sup _{0 \leq \nu \leq 1}|g(y)|\right] .
$$

For such a choice of $\lambda$, one has (11) for all $0 \leq x \leq 1$; hence, application of Theorem 1 yields

$$
\left|v(x)-f(x) e^{-\lambda x}\right| \leq|g(x)| \exp (-\lambda(x-\alpha(x))) V(\alpha(x)), \quad 0 \leq x \leq 1,
$$


where

$$
V(z)=\frac{\sup _{n \leq y \leq z}\left|f(y) e^{-\lambda y}\right|}{1-\sup _{0 \leq \nu \leq z}|g(y) \exp (-\lambda(y-\alpha(y)))|}, \quad 0 \leq z \leq 1 .
$$

Recalling that $u(x)=v(x) e^{\lambda x}$, one obtains:

Theorem 3. Let $f, g$, and $\alpha$ be real and continuous on the interval $0 \leq x \leq 1$. Suppose that $\alpha(0)=0$ and $0 \leq \alpha(x)<x$ for $0<x \leq 1$; assume also that $|g(0)|<1$. Let $u$ be a real and continuous solution of the equation

$$
u(x)=f(x)+g(x) u(\alpha(x)), \quad 0 \leq x \leq 1 .
$$

Then

$$
|u(x)-f(x)| \leq|g(x)| e^{\lambda \alpha(x)} V(\alpha(x)), \quad 0 \leq x \leq 1,
$$

where $V$ is defined by (12),

$$
\lambda=0, \text { if } \sup _{0 \leq \nu \leq 1}|g(y)|<1,
$$

and $\lambda$ satisfies

$$
\lambda>\frac{1}{m} \log \left[\sup _{0 \leq v \leq 1}|g(y)|\right], \quad \text { if } \sup _{0 \leq \boldsymbol{y} \leq 1}|g(y)| \geq 1 .
$$

Again, this bound yields, immediately, the uniqueness of a solution to Eq. (10).

(c) Functional-integral Equations. As a further illustration of the procedure given above, one can consider a linear combination of the operators defined by the two preceding examples. Namely, consider the functional-integral equation

$$
u(x)=f(x)+g(x) u(\alpha(x))+\int_{0}^{x} k(x, y) u(y) d y, \quad 0 \leq x \leq 1,
$$

where $f, g$, and $\alpha$ are as in example (b), and $k$ is real and continuous on $0 \leq y \leq x \leq 1$. In this case, one may take $S=[0,1], S_{0}(x)=S_{1}(x)=[0, x]$, and

$$
T\left(x ; u(s), s \in S_{0}(x)\right)=g(x) u(\alpha(x))+\int_{0}^{x} k(x, y) u(y) d y,
$$

for all $0 \leq x \leq 1$. Again, consider the equation satisfied by the function defined by $v(x)=K^{-1} u(x)=e^{-\lambda x} u(x), 0 \leq x \leq 1$, where $u$ is assumed to be a solution of (13) and $0<\lambda$. That is,

$$
v(x)=f_{1}(x)+T_{1}\left(x ; v(s), s \in S_{0}(x)\right), \quad 0 \leq x \leq 1,
$$

where $f_{1}(x)=e^{-\lambda x} f(x)$ and

$$
T_{1}(x ; v(\cdot))=g(x) \exp (-\lambda(x-\alpha(x))) v(\alpha(x))+e^{-\lambda x} \int_{0}^{x} k(x, y) e^{\lambda \nu} v(y) d y,
$$

for all $0 \leq x \leq 1$.

The mapping $T_{1}$ will satisfy the "size" restriction of Theorem 1 if $\lambda$ is chosen so large that

$$
|g(x)| \cdot \exp (-\lambda(x-\alpha(x)))+\sup _{0 \leq \boldsymbol{\nu} \leq x}|k(x, y)| \cdot \frac{1-e^{-\lambda x}}{\lambda}<1
$$


for all $0 \leq x \leq 1$. It is clear that this condition can be satisfied for all $\lambda$ sufficiently large. A condition which is sufficient for (14) to hold will now be derived. Let $\rho$ be such that $|g(0)|<\rho<1$; let $\delta$ be such that $0<\delta \leq 1$ and

$$
|g(x)| \cdot \exp (-\lambda(x-\alpha(x)))<\rho
$$

for all $0 \leq x \leq \delta$; and let $m=\min _{\delta \leq v \leq 1}(y-\alpha(y))$. Suppose $\lambda$ satisfies

$$
\lambda>\max \left[\frac{1}{m} \log \left(\frac{\sup _{0 \leq \nu \leq 1}|g(y)|}{\rho}\right), \frac{1}{1-\rho} \sup _{0 \leq \nu \leq x \leq 1}|k(x, y)|\right] .
$$

(1) Then, for $0 \leq x \leq \delta$, one has

$$
|g(x)| \cdot \exp (-\lambda(x-\alpha(x)))+\sup _{0 \leq \nu \leq x}|k(x, y)| \cdot \frac{1-e^{-\lambda x}}{\lambda}<\rho+\left(\sup _{0 \leq \nu \leq x \leq 1}|k(z, y)|\right) / \lambda<1,
$$

as desired.

(2) Next, if $\delta \leq x \leq 1$, then

$$
\begin{aligned}
|g(x)| \cdot \exp (- & \lambda(x-\alpha(x)))+\sup _{0 \leq \nu \leq x}|k(x, y)| \cdot \frac{1-e^{-\lambda x}}{\lambda} \\
& \leq \sup _{0 \leq \nu \leq 1}|g(y)| \cdot e^{-\lambda m}+\left(\sup _{0 \leq \nu \leq x \leq 1}|k(z, y)|\right) / \lambda<\rho+\left(\sup _{0 \leq \nu \leq z \leq 1}|k(z, y)|\right) / \lambda<1,
\end{aligned}
$$

which shows (15) to be the desired sufficient condition for (14) to hold.

If $\lambda$ is such that (14) holds for all $0 \leq x \leq 1$, then Theorem 1 may be applied, to obtain

$$
\begin{aligned}
&\left|v(x)-f_{1}(x)\right| \leq|g(x)| \exp (-\lambda(x-\alpha(x))) V(\alpha(x))+e^{-\lambda x} \int_{0}^{x}|k(x, y)| e^{\lambda \nu} V(y) d y, \\
& 0 \leq x \leq 1,
\end{aligned}
$$

where

$$
V(x)=\frac{\sup _{0 \leq \nu \leq x}\left|f(y) e^{-\lambda \nu}\right|}{1-\sup _{0 \leq \nu \leq x}\left[|g(y)| \exp (-\lambda(y-\alpha(y)))+e^{-\lambda \nu} \int_{0}^{\nu}|k(y, z)| e^{\lambda z} d z\right]}
$$

for $0 \leq x \leq 1$. Upon recalling that $u(x)=v(x) e^{\lambda x}, 0 \leq x \leq 1$, one obtains:

Theorem 4. Let $f, g, \alpha$, and $k$ be as specified above. Then, if a continuous solution. $u$ of Eq. (13) exists, it must satisfy, for all $0 \leq x \leq 1$ :

$$
|u(x)-f(x)| \leq|g(x)| e^{\lambda \alpha(x)} V(\alpha(x))+\int_{0}^{x}|k(x, y)| e^{\lambda \nu} V(y) d y,
$$

where $V$ is defined by (16),

$$
\lambda=0, \quad \text { if } \sup _{0 \leq \boldsymbol{y} \leq 1}\left[|g(y)|+\int_{0}^{\boldsymbol{v}}|k(y, z)| d z\right]<1,
$$

and $\lambda$ satisfies (15) [a fortiori (14)] otherwise. 
As before, the bound yields the uniqueness of a solution to Eq. (13). Finally, it should be mentioned that systems of the type (10) and (13) arise in the theory of certain boundary value problems for hyperbolic partial differential equations; see, for example, Picard [3], Chu and Diaz [2], and Chu [4].

\section{REFERENCES}

[1] A. P. Balachandran, Inequalities for the solutions of linear integral equations, J. Math. Phys. 6, 15301533 (1965)

[2] S. C. Chu and J. B. Dian, A fixed point theorem for "in the large" application of the contraction principle, Atti Acad. Sci. Torino 99, 351-363 (1964-65)

[3] E. Picard, Lȩons sur quelques types simples d'equations aux dérivées partielles avec des applications a la physique mathematique, Gauthier-Villars, Paris, 1927

[4] S. C. Chu, On a mixed boundary value problem for linear third order hyperbolic partial differential equations, Rend. Circ. Mat. Palermo, Ser II, 13, 199-208 (1964) 